\title{
Modified Curvature-based Trigonometric Identities for Retinal Blood Vessel Tortuosity Measurement in Diabetic Retinopathy Fundus Images
}

\author{
N. Badariah A. Mustafa ${ }^{1}$, W. Mimi Diyana W. Zaki ${ }^{1 *}$, Aini Hussain ${ }^{1}$, Jemaima Che Hamzah ${ }^{2}$ \\ ${ }^{I}$ Centre for Integrated System Engineering and Advanced Technologies (INTEGRA), Faculty of Engineering and Built Environment, \\ Universiti Kebangsaan Malaysia, Bangi, Selangor, Malaysia \\ ${ }^{2}$ Ophthalmology Department, Medical Faculty, Universiti Kebangsaan Malaysia Medical Centre (UKMMC), Bandar Tun Razak, Che- \\ ras, Kuala Lumpur, Malaysia \\ *Corresponding author E-mail: wmdiyana@ukm.edu.my
}

\begin{abstract}
In current clinical practice, there is no specific standard and grading system that can be used to measure the behaviour of the retinal blood vessel curvature. The retinal blood vessel curvature is measured based on clinical experiences. It is very subjective and inconsistent to describe the presence of tortuosity in fundus images. Thus, this paper aims to measure the tortuosity of retinal blood vessel using curvature-based method and investigate its relationship with diabetic retinopathy (DR) disease. The proposed tortuosity measures have been tested on 43 fundus images belonging to patients who have been diagnosed with DR disease and validated by two clinical experts from our collaborative hospital. On average, the proposed algorithm achieved 90.7\% (accuracy), 98.72\% (sensitivity) and 9.3\% (false negative rate), that shows significant tortuosity presence in diabetic retinopathy fundus images.
\end{abstract}

Keywords: Tortuosity; Curvature-based Method; Retinal Blood Vessel; Diabetic Retinopathy; Digital Fundus Images.

\section{Introduction}

Diabetic eye disease consists of a group of eye conditions that affect people with diabetes, such as diabetic retinopathy (DR), diabetic macular oedema (DME), cataract and glaucoma. DR evolves with changes in blood vessel structures, thereby leading to bleeding or leaking fluid and distort vision. DR is also the most common cause of vision loss among diabetic patients. In Malaysia, according to the National Health and Morbidity survey 1986 and the Global Non-Communicable Disease Report 2014, the number of diabetic patients shows significant increase every year. The number of Malaysians diagnosed with diabetes has increased to $15.2 \%$ in 2011 from $11.6 \%$ in 2006 and it kept increasing to $17.5 \%$ (2015) [1]. This percentage is considered high compared with countries in Asia, such as China $(9.2 \%)$ and India $(8.6 \%)$. Based on the latest statistic in 2015, 3.5 million Malaysian adults are diagnosed with diabetes, and more than half of the numbers (9.2\%) do not know they have the disease. This amount is very worrying, because it shows lack of awareness about this disease among Malaysians. It is important for diabetic patients to go for DR screening process through eyes examination [2]. If left untreated, diabetes will lead to worse DR stage and may cause permanent blindness [3-5].

Thus, these pathologies need to be diagnosed and treated effectively at an early stage, during which accurate and objective analyses are performed. The analyses should be sensitive to detect minor pathological signs; they should also be robust and comparable with accepted clinical standards and consistent with results obtained from the same patient at different times [6]. This work sought to investigate the association of retinal vascular tortuosity with DR using proposed quantitative measurement algorithm. The evaluation of tortuosity is readily accessible in the confined region of a retinal fundus photograph. In current clinical evaluation (Early Treatment Diabetic Retinopathy Study, ETDRS), tortuosity is not critically used when evaluating diabetic fundus, especially for retinopathy cases [7]. However, in a number of pathologies and genetic disorders such as systemic hypertension, long-term diabetes, diabetic retinopathy and disease in retinopathy of prematurity (ROP) [8-11], these diseases had strong correlations with tortuosity. Thus, tortuosity has been proposed to be included in disease assessment as an additional marker for the identification of diabetes besides the existing characteristics of ETDRS [8, 12].

A normal retinal blood vessel structure can be recognised as smooth curvy-like vessel branches, as shown in Fig. 1(a). However, Fig. 1(b) shows the abnormal retinal blood vessel structure that consist of tortuous vessels. The character of the curves is measured using curvature mathematical tool to quantify the inflection of a curve at a certain coordinate [13]. Tortuosity is a curve property that describes the curve's twistedness or crookedness. It is defined as twisted appearance of retinal vascular compare to normal blood vessels that usually appeared straight or gently curve [14-15]. In addition, tortuosity was introduced as one of the measured characteristics besides the existing abnormal features such as exudate, microaneurysm, cotton wool spot and haemorrhage [1618]. Curvature-based method is the most common method used in measuring tortuosity because of its reliability in computation [8], [13, 19-22]. However, it imposes heavy computational burden compared with the arc-to-chord length ratio method. Thus, a template disc approach was applied in curvature estimation, to provide a relatively low computational complexity [13]. 


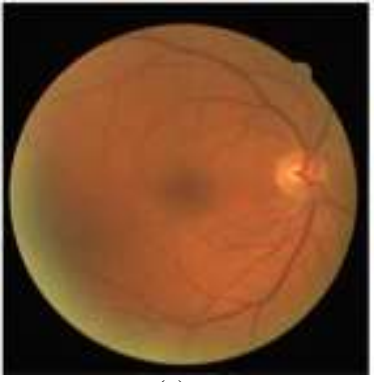

(a)

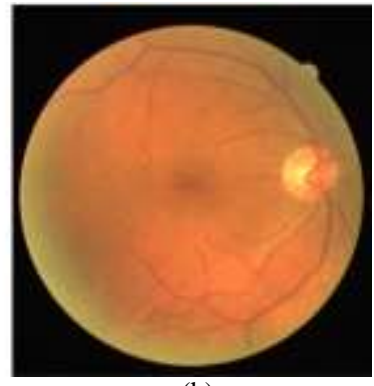

(b)
Fig. 1: Retinal blood vessel structure: (a) Normal curvy-like vessels structure; (b) Abnormal structure (consist of tortuous vessels).

We proposed the tortuosity measurement method using the modified curvature-based method that applied template disc approach and trigonometric identities to curvature function. This measurement is tested on 43 DR images from DRIVE [23], STARE [24] and HRF [25] databases. The tortuosity value obtained from proposed algorithm is compared with a predefined threshold value in order to identify the DR image in a DR with tortuous vessels or a DR with non-tortuous vessels. Then correlation between retinal blood vessel tortuosity and DR will be identified. This will significantly contribute to the practice of considering tortuosity as one of the important criteria in measuring DR progression. The rest of this paper is organised as follows. Section II describes the methods used and the proposed algorithm to measure the retinal blood vessel tortuosity. Results with detail discussions are provided in Section III together with a comparison between previous and proposed tortuosity measure. Finally, Section IV provides the conclusions of this paper.

\section{Literature Review}

In current process, DR is diagnosed through patients' fundus images and the presence of DR abnormal features such as exudates, haemorrhage, cotton wool spots and microaneurysms are manually identified by clinical experts [26]. However, this manual process is a tedious work, tiring, time consuming and imposes high workloads to ophthalmologists. The shortage of professional clinical observers and expert ophthalmologists has encouraged the usage of computer-assisted monitoring. In addition, the consequence of manual process is a long waiting time for patients to receive the diagnosis results and it leads to late treatments. Hence, an automated DR grading system is developed by researchers to aid the ophthalmologists in this time consuming screening and grading process [27-28]. An automated DR screening and grading system is needed because the number of diabetic patients is increasing every year, and it has become a major challenge for health care provider to come up with a systematic and time saving system to accurately diagnose DR in diabetic patients. In [28] have proposed a study on the efficiency of classifying disease or no disease conditions, in which a comparison is made between manual and automated grading system and against the reference standard. This study offers more sensitive but less specific system compared with a manual system, and it could reduce the grading workload required. Furthermore, an automated grading system is operated at a lower cost and is similarly effective when compared with a manual grading system $[27,29]$. However, the cost effectiveness of an automated grading system is less favourable if the area lacks infrastructure such as in rural areas [29].

Tortuous blood vessels happen when the blood vessels are dilated, thereby causing twisting and turning in their direction. Dilation is caused by radial stretching of the blood vessel, and twisting and turning occur due to longitudinal stretching [30]. Tortuosity may occur in a small region of retinal blood vessel or may also involve the entire retinal vascular network [30]. Tortuosity is linked with $\mathrm{DR}$, as it increases with diabetic progression levels. This finding has been reported by several studies [14, 31-35]. Nevertheless, in other studies, inconsistent findings have been reported on the relationship between tortuosity and DR. A strong association of tortuosity and DR is found in patients with longer diabetes duration and type 2 diabetic patients with No DR [16, 36]. In [36] found a $26 \%$ increase of average arteriovenous channels in type 2 diabetes mellitus with no DR subjects. In an Asian population, Cheung et al. concluded that persons with diabetes have less tortuous arteriole than without diabetes; among non-diabetic subjects, those with retinopathy have more tortuous venules than those without retinopathy [10]. Thus, research on the association between retinal vascular tortuosity and DR is still in its infancy. An objective and reliable tortuosity index is needed in order to support early hypotheses.

A precise guideline and standard description of abnormal retinal blood vessel tortuosity has not been formally established. Usually, ophthalmologists will describe and evaluate the blood vessel tortuosity based on their experience. It is done by recognising relative characteristics such as differences of abnormal vessels to normal healthy vessels in terms of vessel's length, width, location and type and number of twists [37]. Therefore, researchers need to develop mathematical formulations of tortuosity (quantitative measurement) to meet the needs of ophthalmologists'. Quantitative measurement of retinal blood vessel tortuosity was first introduced in 1979 by [38]. They presented the idea of tortuosity by measuring the relative length of vessel curve over the length between two end points; this is known as arc-to-chord ratio method. This method was also adapted by other researchers [39-45] because it is the simplest method for measuring retinal blood vessel tortuosity. However, this method only compares vessel elongation without considering the shape of the vessel [4]. Furthermore, using this method, the curve segment (non-tortuous) and sinusoidal segment (tortuous) that have the same arc and chord lengths leads to the same tortuosity value $[4,46]$.

The limitation found in terms of angle changes due to vessel direction and has driven the formulation of an angle variation method [31, 34, 46-47]. However, it does not consider the shape of the vessel curves, and the vessel segments with no variation in direction will not contribute to tortuosity measure. Other domain-based methods for evaluating blood vessel tortuosity have been introduced by other studies [48-50]. However, these proposed methods are difficult to apply and highly sensitive to noise during vessel extraction to obtain vessel midline [48]. Furthermore, lower accuracy was achieved when compare to arc-to-chord ratio in terms of Spearman correlation [50]. Hence, we proposed a quantitative measurement that makes use of modified curvature-based trigonometric identities. This measurement applied the modified template disk method and trigonometric identities to extended curvature function to measure the vessel curvature. The proposed measures identified the curve vessel as tortuous if the curve value is lower than a predefined threshold value, as described in Section 3.

\section{Methodology}

This work is conducted due to early hypotheses emphasising that tortuosity of retinal blood vessel in patients with DR is higher than in normal patients and increases with DR severity. Initially, the diagnosed DR's image is assumed to contained tortuous vessels. The measurement of retinal blood vessel tortuosity requires blood vessel segmentation, vessel thinning process, detection and removal of blood vessel crossover and bifurcation nodes. Lastly, the local and global tortuosity measurements are performed. The process began by manually sorting the fundus images of the right and left eyes. Then, the fundus image (RGB image) undergoes the segmentation phase to obtain the retinal blood vessel network, and the unwanted noises (artefacts) and lesions are removed. Retinal blood vessel segmentation is a preliminary step for most vessel analyses procedures, such as measurement of vessel tortuosity, vessel width, vessel length, vessel diameter and others. In the 
retinal blood vessel segmentation process, the blood vessel networks were classified into vessel and non-vessel pixels. Next, the thin branches of retinal blood vessel were removed from the structure, thereby leaving only the main and first branches (thick branches). In current practices, clinical graders evaluate the retinal blood vessel on the thick branches, as it appears more visible to the naked eye compared with thin branches. Thus, the retinal blood vessel tortuosity was measured on the thick branches, so that our findings will be in line with those of clinical graders.

The tortuosity of the blood vessel was measured using the proposed modified curvature-based method that applied the modified template disk approach and trigonometric identities to extended curvature function. Fig. 2 depicts the flow chart of the proposed method, which is discussed in detail in the following subsections.

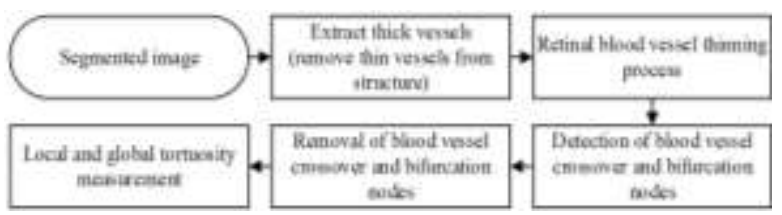

Fig. 2: Flow chart of the proposed method for retinal blood vessel tortuosity measurement

\subsection{Retinal Blood Vessel Segmentation and Thick Vessels Extraction}

In this subsection, the segmentation process was adapted from previous studies [51-52] with some improvements in the preprocessing stage. Filling algorithm is added to this pre-processing stage in order to fill the vessel's silver and copper wiring and the vessel central reflex on the homogenised image. The filling algorithm applied the morphological operations, namely closing and median filtering operation, to the homogenised image. The implementation of retinal blood vessel segmentation algorithm on the fundus image is described in Fig. 3. The thick vessels were extracted from the segmented image using a morphological operation that involved erosion operation and removal of segments that is less than 100 pixels in length. Fig. 4 illustrates an example of original fundus image, segmented image and extracted thick vessels.

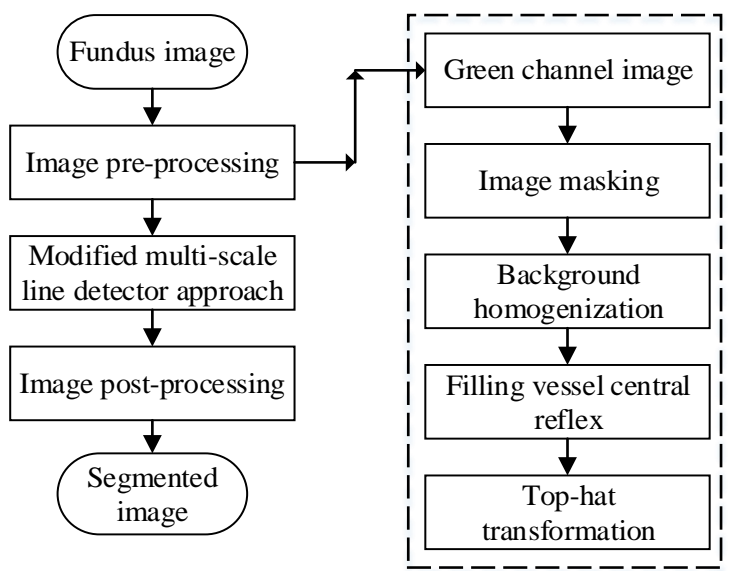

Fig. 3: Flow chart of implementation of retinal blood vessel segmentation algorithm on fundus image

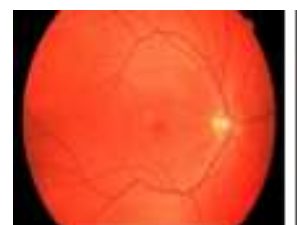

(a)

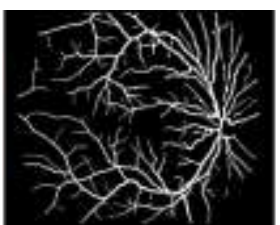

(b)

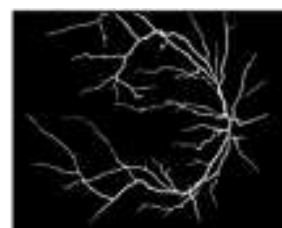

(c)
Fig. 4: Retinal blood vessel segmentation and extraction of thick vessels (a) Original fundus image; (b) Segmented image; (c) Extracted thick vessels

\subsection{Tortuosity Pre-Processing}

This stage consists of several processing steps before blood vessels' tortuosity can be measured. This stage includes blood vessel thinning process, detection of crossover and bifurcation nodes and removal of such nodes from retinal blood vessel segment. Retinal blood vessel thinning process is performed using morphological thinning algorithm, bwmorph, as suggested by [53]. Then, the crossover and bifurcation nodes are identified from the skeletonised blood vessel tree. In order to identify the nodes, it is important to understand the definition of crossover and bifurcation junctions. Crossover is defined as a point of crossing from one side to the other, which causes the vessel to overlap with other vessels. Bifurcation is defined as the division of one vessel branch into two vessel branches. The definition of these junctions is illustrated in Fig. 5.

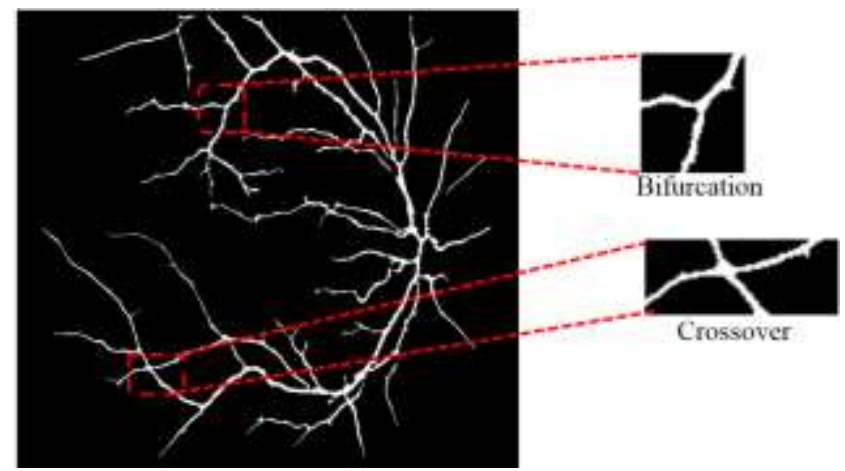

Fig. 5: Illustration of bifurcation and crossover junctions

In the next process, the segmented image that contained only thick vessels went through the thinning process to obtain the skeletonised retinal blood vessel branches. The bifurcation and crossover nodes were then identified from the skeletonised structure shown in Fig. 6(a). The identified nodes were then extracted from the blood vessel structure, thereby leaving only the blood vessel subsegments. The sub-segments are shown in Fig. 6(b). The purpose of segregating the vessel segment into sub-segments was easily measure local tortuosity.

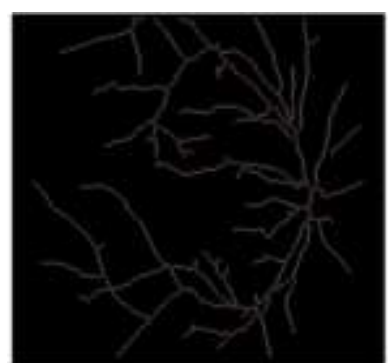

(a)

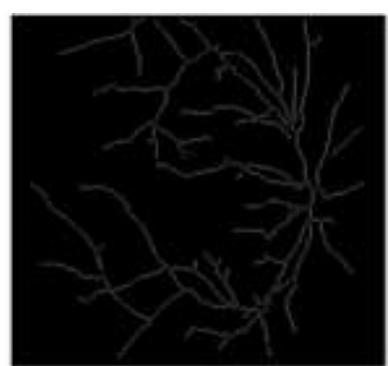

(b)
Fig. 6: Skeletonised segmented image: (a) Identified bifurcation and crossover nodes; (b) sub-segments on retinal blood vessel structure

\subsection{Tortuosity Measurement using Modified Curva- ture-based Method}

In this work, the proposed retinal blood vessel tortuosity measurement used the combination of local and global measurements adopted from [54, 13]. The local measurement computes the tortuosity value for each sub-segment, and each sub-segment values will be added and averaged to obtain the global tortuosity value. In (1) shows the mathematical expression of global retinal blood vessel measurement, as follows:

$\tau=\frac{1}{m} \sum_{i=1}^{m} k_{i}$ 
where $\mathrm{k}_{\mathrm{i}}$ denotes the local tortuosity measure of $\mathrm{i}$-th sub-segment point or line on the blood vessel structure and $\mathrm{m}$ is the total number of sub-segment point or line in the structure. Basically, global measurement can be said as the average value of the blood vessel segments in the fundus image.

In order to locally measure the vessel sub-segments, this work proposed a quantitative measurement used modified curvaturebased trigonometric identities. This measurement applied the modified template disk method and trigonometric identities to extended curvature function to measure the vessel curvature. In (2) presents a planar curve of a continuous case, $y=f(x)$, which can be written as follows:

$$
k=\frac{y^{n}}{\left(1+y^{s 2}\right)^{\frac{3}{2}}}
$$

The parametric representation of a curve with $x=x(t)$ and $y=y(t)$ is shown in (3).

$k=\frac{y^{s t}(t) x^{t}(t)-x^{t v}(t) y^{\prime \prime}(t)}{\left(x^{\prime}(t)^{2}+y^{\prime}(t)^{2}\right)^{\frac{3}{2}}}$

A template disk with a suitable radius is drawn on the curve and produces overlapping region between them. The overlapping region is represented by a point at zero first derivative as follows:

$k=f^{n s}(x)$

Hence, the curvature function using Taylor series for points in the vicinity of zero [9] is defined as follows:

$$
f(x)=\frac{1}{2} k x^{2}+o\left(x^{3}\right)
$$

where $\mathrm{k}$ denotes the curvature at the origin, and $\mathrm{o}\left(\mathrm{x}^{3}\right)$ expresses the higher order term of the series. Applying polar coordinates ( $\mathrm{r}$, $\theta$ ) to planar curve, $y=f(x)$, we acquired the following:

$$
r \sin \theta \approx \frac{1}{2} k r^{2} \cos ^{2} \theta+r^{3} \cos ^{3} \theta
$$

Then, the coordinate systems in (6) is normalised by the radius of template disk, $\mathrm{b}$, in which $\mathrm{R}=\mathrm{r} / \mathrm{b}$ and $\mathrm{K}=\mathrm{kb}$. In this case, we assume that the radius of template disk is $b=1$ since this value provides the ideal template disk diameter.

$$
R \sin \theta \approx \frac{1}{2} K R^{2} \cos ^{2} \theta+R^{3} \cos ^{3} \theta
$$

Next, $\theta_{c}$ is derived by setting $R=1$ in (7). Thus, we obtained the following:

$$
\begin{aligned}
& \sin \theta_{c} \approx \frac{1}{2} K \cos ^{2} \theta_{c}+\cos ^{3} \theta_{c} \\
& \frac{1}{2} K \cos ^{2} \theta_{c} \approx \sin \theta_{c}-\cos ^{3} \theta_{c} \\
& K \approx \frac{2 \sin \theta_{c}}{\cos ^{2} \theta_{c}}-\frac{2 \cos ^{3} \theta_{c}}{\cos ^{2} \theta_{c}}
\end{aligned}
$$

The derived relation between $\mathrm{K}$ and $\theta_{\mathrm{c}}$ is stated in (11).

$$
K \approx \frac{2 \sin \theta_{c}}{\cos ^{2} \theta_{c}}-2 \cos \theta_{c}
$$

Applying the trigonometric identity $\cos ^{2} \theta_{c}=1-\sin ^{2} \theta_{c}$ and $K=$ $\mathrm{kb}$ in (11), we finally obtained the curvature function in the form of $\mathrm{k}$

$k \approx \frac{2 \sin \theta_{c}}{b\left(1-\sin ^{2} \theta_{c}\right)}-\frac{2 \cos \theta_{c}}{b}$

The proposed tortuosity measurement $\left(\mathrm{k}_{\mathrm{tr} \_ \text {modified }}\right)$ is defined as follows:

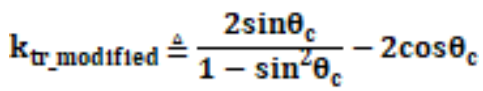

The proposed method is resilient against noise and variant to translation, rotations and scaling and returned milder results, as shown in Table 1 . The advantage of $\mathrm{k}_{\text {tr_modified }}$ is this method does not ignore the highest order of the curvature function $\left(\mathrm{R}^{3} \cos ^{3} \theta\right)$ as it presents the complete cycle of the curve. The order significantly indicates the curve's shape and direction. Therefore, it is beneficial to implement this method in measuring the tortuosity of retinal blood vessels. As shown in Table 1, $\mathrm{k}_{\text {tr_modified }}$ measurement has been conducted on three synthetic images. The curvature value is decreasing as the curve became curvier or more tortuous.

\begin{tabular}{|l|c|c|}
\hline \multicolumn{1}{|c|}{ Table 1: $\mathrm{k}_{\mathrm{tr} \text { modified }}$ value of curve synthetic images } \\
\hline No. & Synthetic Image & $k_{\text {tr modified }}$ \\
\hline & & \\
\hline & & \\
\hline & & \\
\hline
\end{tabular}

\subsection{Tortuosity Measurement: Parameter Setting}

As mentioned in previous sub-sections, tortuosity is measured locally using modified curvature-based method and globally by finding the average tortuosity value for the whole sub-segments in the quadrant. In order to classify the DR image in an image with tortuous vessels or with non-tortuous vessels, a threshold value is determined in (14) as follows:

Threshold $=\frac{\tau_{\text {maximum }}+\tau_{\text {minimum }}}{2}$

where $\tau_{\text {maximum }}$ is the maximum curve value of DR image, and $\tau_{\text {minimum }}$ is the minimum curve value of DR image. The DR image is classified as an image with tortuous vessels if the curve value is less than the threshold and classified as an image with nontortuous vessels if the curve value is greater than the threshold. In the following section, the results obtained from the proposed measurement and validations with two clinical expert graders were discussed in order to determine the effectiveness of the algorithm. Based on the findings, we concluded that a relationship was present between retinal blood vessel tortuosity and DR disease. 


\section{Results and Discussion}

In this work, 43 DR images obtained from DRIVE, STARE and HRF databases were used to evaluate the performance of the proposed tortuosity measurement algorithm. The fundus images were already confirmed to have DR based on the diagnosis provided from the databases. The grading was made upon the appearance of abnormal DR pathological features such as microaneurysms, exudates, haemorrhages and cotton wool spot in the fundus image. Two clinical experts (A and B) were assigned to measure the tortuosity of retinal vessel, because a precise guideline and standard description of abnormal retinal blood vessel tortuosity is not formally established. The retinal vessel tortuosity was observed and evaluated based on the experts' clinical experiences. From the observation, Expert A has classified 39 and 4 images to be DR with tortuous vessels (DRWT) and DR without tortuous vessels (DRWoT), respectively. Meanwhile, Expert B classifies 37 images as DRWT and 6 images as DRWoT. Thus, in overall it can be concluded that both experts have almost similar prediction outcomes for all images in which their outcomes were in agreement.

Table 2 contains four $\tau_{\text {tr_modified }}$ values for the selected DR images. The images were classified into two categories, namely, DRWT and DRWoT. $\tau_{\text {tr_modified }}$ is determined based on the average curve value of main and first branches. From Table 2, average curve value, which is less than the threshold value was categorised as DRWT and vice versa. The threshold value was determined in (14). Segmented image numbers 2 and 4 still consisted of unwanted noise (exudates and laser marks) that were difficult to remove by segmentation process. These noises were classified as false negative (FN) components.

Table 2: $\tau_{\text {tr_modified }}$ value for selected DR images

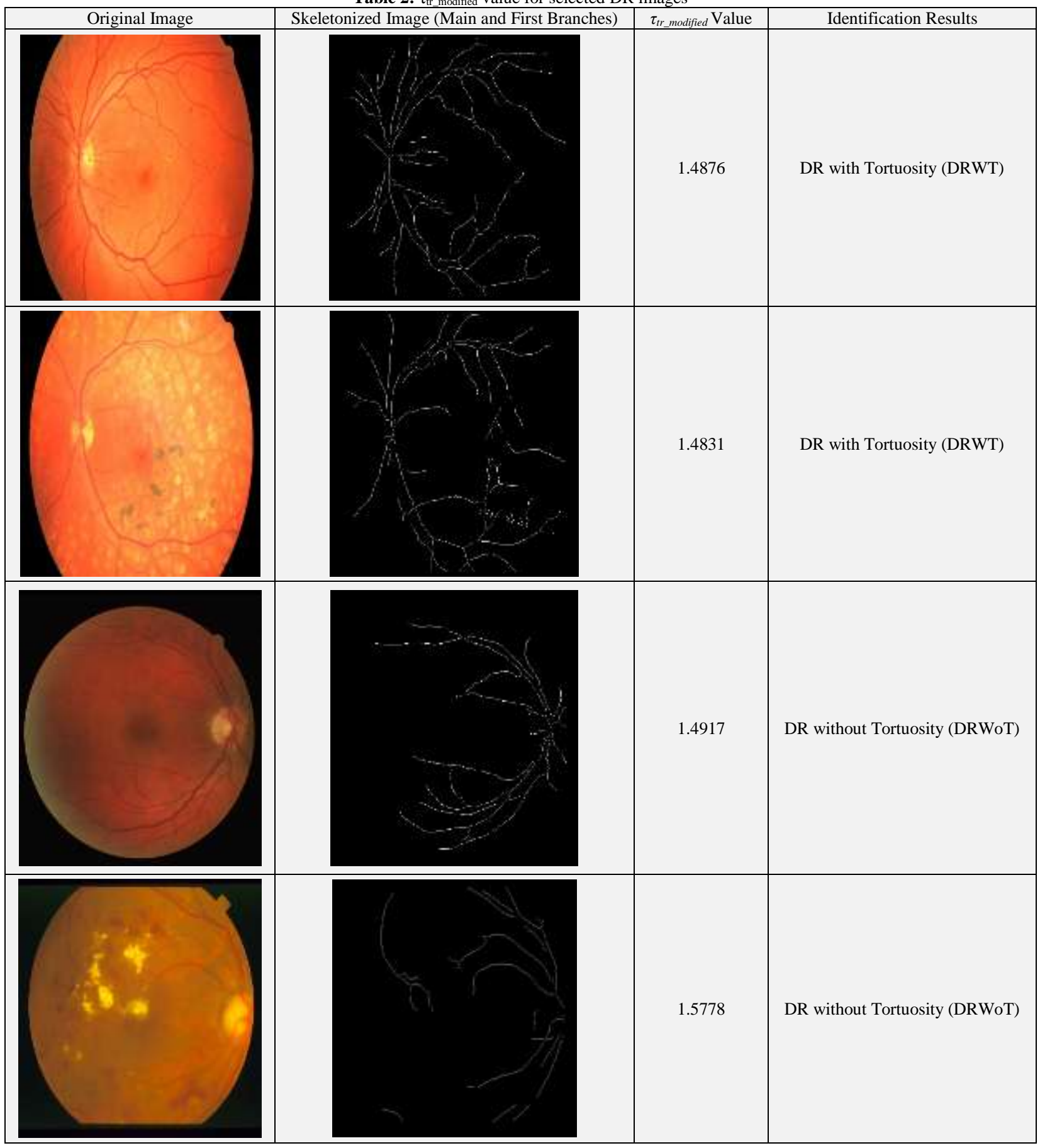


The performance of proposed algorithm in measuring the blood vessels tortuosity of DR images was evaluated by its application to $43 \mathrm{DR}$ images. The goal of the evaluation was to determine the positive cases of DR that consisted of tortuous vessels. Two ophthalmologists (clinical experts) on two different occasions were asked to give their observation on existence of tortuosity in the DR fundus images. The tortuosity was evaluated by assigning ' $T$ ' and ' $\mathrm{N}$ ' to the positive (tortuous) and the negative (non-tortuous) cases respectively. Then, the proposed algorithm was used to measure tortuosity of retinal blood vessel in the fundus image. The outcomes obtained from the proposed algorithm were compared with the evaluation made by two clinical experts. Fig. 7 shows the performance measures of $\tau_{\text {tr_modified }}$ on 43 DR images. In medical diagnosis, sensitivity and specificity are useful for evaluating a clinical test, whereas positive and negative predictive values (PPV and NPV) are useful in disease screening or preclinical tools. PPV and NPV are dependent on the prevalence of the disease in a population of interest; in this paper, we focus on DR population. The predictive value provides an answer to clinicians who evaluate the chances of diabetic patient to develop DR disease, even if the test result is positive. Meanwhile, the percentage of accuracy represents the evaluation of the accuracy of a proposed algorithm quality.

The overall performance of the proposed algorithm can be seen from the percentage of accuracy. The proposed algorithm achieved $90.7 \%$ accuracy (Acc) based on the validation of two clinical experts. Higher sensitivity (Sen) values $(98.72 \%$ on average) and lower specificity (Spe) values (29.17\% on average) showed that there are few false negative (FN) results and thus, fewer cases of disease missed. Higher PPV value $(91.46 \%$ on average) shows that the proposed algorithm provided a high probability of subjects with a positive screening test to develop the disease. Meanwhile, NPV percentage (75\% on average) projected a good probability of identifying subjects without disease from a negative screening test. The low false negative rate, FNR $(9.3 \%)$ demonstrated the low disagreement between proposed algorithm outcomes and clinical experts' judgement. The performance measures outcome indicated the significant agreement among experts and proposed algorithm outcomes, $\tau_{\text {tr modified }}$ (see Fig. 7). It also showed a strong correlation, which manifested the capability of the proposed algorithm to determine the positive cases of tortuosity in DR fundus images.

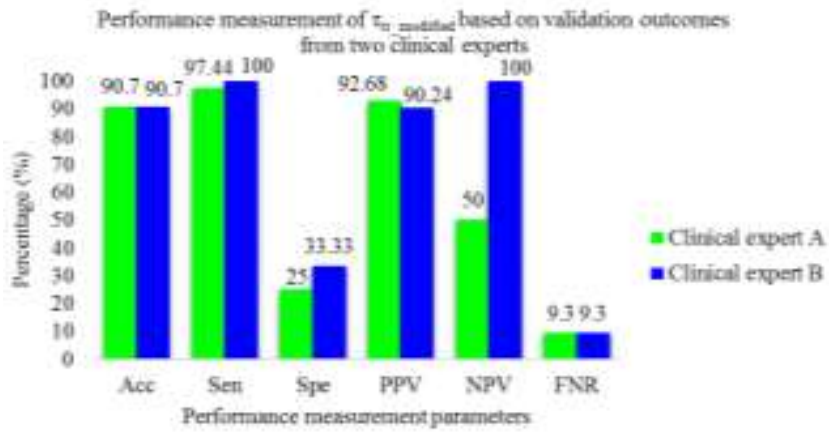

Fig. 7: Performance measurement of $\tau_{\text {tr_modified }}$ based on validation outcomes from two clinical experts

\section{Conclusion}

A quantitative approach that used the modified curvature-based method and trigonometric identities was proposed to measure the tortuosity of retinal blood vessel in DR fundus images. The proposed algorithm applied the modified template disk and trigonometry identities to extended curvature function. The algorithm was applied to the selected blood vessels comprising the main and first branches of blood vessels, which are also known as thick vessels.
The results obtained from proposed algorithm were then validated by two clinical experts to determine the correlation between blood vessels tortuosity and DR disease. Excellent matching between algorithm outcomes and additional low FNR (as judged by the experts) showed the significant correlation between tortuosity of retinal blood vessel and DR disease. This finding proved that the tortuosity measurement using proposed algorithm has the bright potential to be used during disease assessment, especially as an additional marker when identifying DR among diabetic patients.

\section{Acknowledgement}

This project is supported by the Ministry of Education (MOE) grant under FRGS/1/2015/TK04/UKM/01/3 and Universiti Kebangsaan Malaysia under DIP-2015-012. Special thanks to the Ophthalmology Department, University Kebangsaan Malaysia Medical Centre (UKMMC) for providing the facilities and experts.

\section{References}

[1] Sulaiman L. H. Beban penyakit diabetes: Kos hanya untuk rawatan mencecah RM4 bilion setahun. 2016 , http://www.utusan.com.my/gaya-hidup/kesihatan/beban-penyakitdiabetes-1.208881.

[2] Bernama. Pesakit kencing manis berdepan risiko buta. 2015, http://www.sinarharian.com.my/nasional/pesakit-kencing-manisberdepan-risiko-buta-1.438659.

[3] Kauppi T., Kalesnykiene V., Kamarainen J.-K., L. Lensu, Sorri I., Ranien A., Voutilainen R., Pietila J., Kalviainen H. and Uusitalo H. The DIARETDB1 diabetic retinopathy database and evaluation protocol. Proceedings of the British Machine Vision Conference 2007, pp. 1-10.

[4] W. Mimi Diyana W. Zaki, M. Asyraf Zulkifley, Aini Hussain, W. Haslina W. A. Halim, N. Badariah. A. Mustafa and Lim Sin Ting. Diabetic retinopathy assessment: Towards an automated system. Biomedical Signal Processing and Control 2016, 24, pp. 72-82.

[5] Mustafa Nur Badariah Ahmad, Wan Mimi Diyana Wan Zaki and Aini Hussain. A review on the diabetic retinopathy assessment based on retinal vascular tortuosity. Proceedings of the IEEE 11th International Colloquium on Signal Processing and Its Applications 2015, pp. 127-130.

[6] Grisan E., Foracchia M. and Ruggeri A. A novel method for the automatic grading of retinal vessel tortuosity. IEEE Transactions on Medical Imaging 2008, 27(3), 310-319.

[7] Hirsch I. B., Brownlee M. Beyond hemoglobin A1c - need for additional markers of risk for diabetic microvascular complications. Journal of the American Medical Association 2010, 303(22), 22912292.

[8] Sasongko M. B., Wong T. Y., Nguyen T. T., Shaw J. E., Jenkins A. J. and Wang J. J. Novel versus traditional risk markers for diabetic retinopathy. Diabetologia 2012, 55(3), 666-670.

[9] Kalitzeos A. A., Lip G. Y. H. and Heitmar R. Retinal vessel tortuosity measures and their applications. Experimental Eye Research 2013, 106, 40-46.

[10] Cheung C. Y., Lamoureux E., Ikram M.K., Sasongko M.B., Ding J., Zheng Y., Mitchell P., Wang J.J. and Wong T.Y. Retinal vascular geometry in Asian persons with diabetes and retinopathy. Journal of Diabetes Science and Technology 2012, 6(3), 595-605.

[11] Mapayi T., Tapamo J.-R., Viriri S. and Adio A. Automatic retinal vessel detection and tortuosity measurement. Image Analysis and Stereology 2016, 35(2), 117-135.

[12] Early Treatment Diabetic Retinopathy Study Research Group and others. Grading diabetic retinopathy from stereoscopic color fundus photographs - an extension of the modified Airlie House classification: ETDRS report number 10. Ophthalmology 1991, 98(5), 786806.

[13] Aghamohamadian-Sharbaf M., Pourreza H. R. and Banaee T. A novel curvature-based algorithm for automatic grading of retinal blood vessel tortuosity. IEEE Journal of Biomedical and Health Informatics 2016, 20(2), 586-595.

[14] Dougherty G., Johnson M. J. and Wiers M. D. Measurement of retinal vascular tortuosity and its application to retinal pathologies. Medical and Biology Engineering and Computing 2010, 48(1), 87-. 
[15] Turior R., Onkaew D., Uyyanonvara B. and Chutinantvarodom P. Quantification and classification of retinal vessel tortuosity. Science Asia 2013, 39, 265-277.

[16] Sasongko M. B., Wong T.Y., Nguyen T.T., Cheung C.Y., Shaw J.E., Kawasaki R., Lamoureux E. and Wang J.J. Retinal vessel tortuosity and its relation to traditional and novel vascular risk markers in persons with diabetes. Current Eye Research 2016, 41(4), 551557.

[17] Ding J.; Ikram M. K., Cheung C. Y. and Wong T. Y. Retinal vascular calibre as a predictor of incidence and progression of diabetic retinopathy. Clinical and Experimental Optometry 2012, 95(3), 290-296.

[18] Ikram M. K., Cheung C.Y., Lorenzi M., Klein R., Jones T.L. and Wong T.Y. Retinal vascular caliber as a biomarker for diabetes microvascular complications. Diabetes Care 2013, 36(3), 750-759.

[19] Johnson M. J., Dougherty G. Robust measures of three-dimensional vascular tortuosity based on the minimum curvature of approximating polynomial spline fits to the vessel mid-line. Medical Engineering and Physics 2007, 29(6), 677-690.

[20] Onkaew D., Turior R., Uyyanonvara B., Akinori N. and Sinthanayothin C. Automatic retinal vessel tortuosity measurement using curvature of improved chain code. Proceedings of the International Conference on Electrical, Control and Computer Engineering 2011, pp. 183-186.

[21] Turior R., Uyyanonvara B. Curvature-based tortuosity evaluation for infant retinal images. Journal of Information Engineering and Applications 2012, 2(8), 9-17.

[22] Bribiesca E. A measure of tortuosity based on chain coding. Pattern Recognition 2013, 46(3), 716-724.

[23] Staal J., Abràmoff M. D., Niemeijer M., Viergever M. A. and Van Ginneken B. Ridge-based vessel segmentation in color images of the retina. IEEE Transactions on Medical Imaging 2004, 23(4), 501-509.

[24] Hoover A., Goldbaum M. Locating the optic nerve in a retinal image using the fuzzy convergence of the blood vessels. IEEE Transactions on Medical Imaging 2003, 22(8), 951-958.

[25] Odstrcilik J., Kolar R., Budai A., Horneggar J., Jan J., Gazarek J., Kubena T., Cernosek P., Svoboda O. and Angelopoulou E. Retinal vessel segmentation by improved matched filtering: Evaluation on a new high-resolution fundus image database. IET Image Processing 2013, 7(4), 373-383.

[26] Patil C. M. An approach for the detection of vascular abnormalities in diabetic retinopathy. International Journal of Data Mining Techniques and Applications 2013, 2, 246-250.

[27] Abramoff M. D., Niemeijer M. and Russell S. R. Automated detection of diabetic retinopathy: Barriers to translation into clinical practice. Expert Review on Medical Devices 2010, 7(2), 287-296.

[28] Philip S., Fleming A.D., Goatman K.A., Fonseca S., McNamee P., Scotlang G.S., Prescott G.J., Sharp P.F. and Olson J.A. The efficacy of automated disease/no disease grading for diabetic retinopathy in a systematic screening programme. British Journal of Ophthalmology 2007, 91(11), 1512-1517.

[29] Scotland G. S., McNamee P., Philip S., Fleming A.D., Goatman K.A., Prescott G.J., Fonseca S., Sharp P.F. and Olson J. A. Costeffectiveness of implementing automated grading within the national screening programme for diabetic retinopathy in Scotland British Journal of Ophthalmology 2007, 91(11), 1518-1523.

[30] Hart W. E., Goldbaum M., Côté B., Kube P. and Nelson M. R. Measurement and classification of retinal vascular tortuosity. International Journal of Medical Informatics 1999, 53(2-3), 239-252.

[31] Dougherty G., Johnson M. J. Clinical applications of threedimensional tortuosity metrics. Proceedings of the Volume 6511, Medical Imaging 2007: Physiology, Function, and Structure from Medical Images 2007, pp. 1-9.

[32] Sasongko M. B., Wong T. Y., Nguyen T. T., Cheung C. Y., Shaw J. E. and Wang J. J. Retinal vascular tortuosity in persons with diabetes and diabetic retinopathy. Diabetologia 2011, 54(9), 2409-2416.

[33] Weiler D. L., Engelke C. B., Moore A. L. O. and Harrison W. W. Arteriole tortuosity associated with diabetic retinopathy and cholesterol. Optometry and Vision Science 2015, 92(3), 384-391

[34] Iorga M., Dougherty G. Tortuosity as an indicator of the severity of diabetic retinopathy. In G. Dougherty (Ed.), Medical Image Processing. Biological and Medical Physics, Biomedical Engineering. New York: Springer, 2011, pp. 269-290.

[35] Sasongko M. B., Wong T.Y., Donaghue K. C., Cheung N., Jenkins A.J., Aguirre P.B. and Wang J.J. Retinal arteriolar tortuosity is associated with retinopathy and early kidney dysfunction in type $1 \mathrm{di}$ abetes. American Journal of Ophthalmology 2012, 153(1), 176-183.
[36] Tam J., Dhamdhere K.P., Tiruveedhula P., Manzanera S., Barez S., Bearse Jr M. A., Adams A.J. and Roorda A. Disruption of the retinal parafoveal capillary network in type 2 diabetes before the onset of diabetic retinopathy. Investigate Ophthalmology and Visual Science 2011, 52(12), 9257-9266.

[37] Abdalla M., Hunter A. and Al-Diri B. Quantifying retinal blood vessels' tortuosity. Proceedings of the Science and Information Conference, 2015, pp. 687-693.

[38] Lotmar W., Freiburghaus A. and Bracher D. Measurement of vessel tortuosity on fundus photographs. Albrecht von Graefes Archiv für klinische und experimentelle Ophthalmologie 1979, 211(1), 49-57.

[39] Capowski J. J., Kylstra J. A. and Freedman S. F. A numeric index based on spatial frequency for the tortuosity of retinal vessels and its application to plus disease in retinopathy of prematurity. Retina (Philadelphia, Pa.) 1995, 15(6), 490-500.

[40] Heneghan C., Flynn J., O'Keefe M. and Cahill M. Characterization of changes in blood vessel width and tortuosity in retinopathy of prematurity using image analysis. Medical Image Analysis 2002, 6(4), 407-429.

[41] Swanson C., Cocker K. D., Parker K. H., Moseley M. J. and Fielder A. R. Semiautomated computer analysis of vessel growth in preterm infants without and with ROP. British Journal of Ophthalmology 2003, 87(12), 1474-1477.

[42] Gelman R., Jiang L., Du Y. E., Martinez-Perez M. E., Flynn J. T. and Chiang M. F. Plus disease in retinopathy of prematurity: Pilot study of computer-based and expert diagnosis. Journal of American Association for Pediatric Ophthalmology and Strabismus 2007, 11(6), 532-540.

[43] Gelman R., Martinez-Perez M. E., Vanderveen D. K., Moskowitz A and Fulton A. B. Diagnosis of plus disease in retinopathy of prematurity using Retinal Image multiScale Analysis. Investigative Ophthalmology and Visual Science 2005, 46(12), 4734-4738.

[44] Wallace D. K. Computer-assisted quantification of vascular tortuosity in retinopathy of prematurity (an American Ophthalmological Society thesis). Transactions of the American Ophthalmological Society 2007, 105, 594-615.

[45] Wallace D. K., Freedman S. F. and Zhao Z. Evolution of plus disease in retinopathy of prematurity: Quantification by ROPtool. Transactions of the American Ophthalmological Society 2009, 107, 47-52.

[46] Oloumi F., Rangayyan R. M. and Ells A. L. Assessment of vessel tortuosity in retinal images of preterm infants. Proceedings of the 36th Annual International Conference of the IEEE in Engineering in Medicine and Biology Society 2014, pp. 5410-5413.

[47] Rodriguez Z. M., Kenny P. and Gaynor L. Improved characterisation of aortic tortuosity. Medical Engineering and Physics 2011 33(6), 712-719.

[48] Dougherty G., Varro J. A quantitative index for the measurement of the tortuosity of blood vessels. Medical Engineering and Physics 2000, 22(8), pp. 567-574.

[49] Wert A., Scott S. E. A new approach to measuring tortuosity. Proceedings of the Volume 8315, Medical Imaging 2012: ComputerAided Diagnosis 2012, pp. 1-6.

[50] Chakravarty A., Sivaswamy J. A novel approach for quantification of retinal vessel tortuosity using quadratic polynomial decomposition. Proceedings of the Indian Conference on Medical Informatics and Telemedicine 2013, pp. 7-12.

[51] [51] V Nguyen U. T., Bhuiyan A., Park L. A. F. and Ramamohanarao K. An effective retinal blood vessel segmentation method using multi-scale line detection. Pattern Recognition 2013, 46(3), 703715 .

[52] Hou Y. Automatic segmentation of retinal blood vessels based on improved multiscale line detection. Journal of Computing Science and Engineering 2014, 8(2), 119-128

[53] Guo Z., Hall R. W. Parallel thinning with two-subiteration algorithms. Communications of the ACM 1989, 32(3), 359-373.

[54] Pourreza H. R., Pourreza M. and Banaee T. Simple and efficient method to measure vessel tortuosity. Proceedings of the 3th International Conference on Computer and Knowledge Engineering 2013, pp. 219-222. 